\title{
Gerschgorin Variations of Complex Matrices
}

\author{
Haifeng Sang \\ Department of Mathematics \\ Beihua University \\ Jilin, China \\ e-mail: sanghaifeng2008@163.com
}

\author{
Qingchun Li \\ Department of Mathematics \\ Beihua University \\ Jilin, China \\ Corresponding author : liqingchun01@163.com
}

\begin{abstract}
We present various “additive” sufficient conditions for the nonsingularity of a complex partitioned matrix and an irreducible partitioned matrix. As the application, a new criteria of the positive stable matrix is given. The obtained results generalize some corresponding results.
\end{abstract}

Keywords- Nonsingularity; Gerschgorin disc; Stable matrix

\section{INTRODUCTION}

Matrix theory which is a fundamental tool in Mathematics has been an important tool in some technologic fields. And estimate of eigenvalue which is a significant part in Matrix theory plays an important role in some relevant fields such as Statistics, Numerical analysis, System engineering, Stability theory,Computation methods, etc.

In this paper, first of all, we discussed the problem of nonsingularity of complex partitioned matrices, gave the conditions of complex partitioned matrices and irreducible partitioned matrices.In addition, we obtained the new criteria for a matrix to be a positive stable matrix. Therefore,we provide a new method solving differential equations in vibrating systems by these results.

\section{NOTATIONS}

Some basic definitions can be found in [5,6,7]. We assume $\langle m\rangle=\{1,2, \cdots, m\}$ and $|S|$ denotes the number of elements in $S$ which is the subset of $\langle m\rangle$. And $A=\left(a_{i j}\right) \in C^{n \times n}$ is partitioned as follows:

$$
A=\left(\begin{array}{cccc}
A_{11} & A_{12} & \cdots & A_{1 m} \\
A_{21} & A_{22} & \cdots & A_{2 m} \\
\vdots & \vdots & \ddots & \vdots \\
A_{m 1} & A_{m 2} & \cdots & A_{m m}
\end{array}\right)
$$

Let $\tilde{R}_{i}(A)=\sum_{j \neq i}\left\|A_{i i}^{-1}\right\| \cdot\left\|A_{i j}\right\|, \tilde{C}_{i}(A)=\sum_{i \neq j}\left\|A_{j j}^{-1}\right\| \cdot\left\|A_{j i}\right\|$, and

$\tilde{C}_{i k}(A)=\max _{|T|=k-1} \sum_{\substack{j \in T \\ j \neq i}}\left\|A_{j j}^{-1}\right\| \cdot\left\|A_{j i}\right\|, i \in<m>$.

\section{MAIN RUSULTS}

Theorem 1. Let $A=\left(a_{i j}\right) \in C^{n \times n}$ be partitioned as (1), in which $A_{i i}$ is nonsingular for $i \in<m>$.If there exists $k \in<m>$ such that
$\left|S_{k}\right|=k$ implies $\sum_{i \in S_{k}}\left(1-\tilde{R}_{i}(A)\right)>0$ for all $S_{k} \subseteq<m>$

and

$$
|S|>\min \left\{\sum_{i \in S} \tilde{R}_{i}(A), \sum_{i \in S} \tilde{C}_{i k}(A)\right\} \text { for all } S \subseteq<m>\text {. }
$$

Then $A$ is nonsingular.

Proof. Assume the hypotheses of the theorem, and $A$ is singular. Then there exists a nonzero vector $Z$ such that $A Z=0$, in which the partitioned vector of $Z$ is uniform with $A$. Without loss of generality, we assume that $X_{j}=\left\|Z_{j}\right\|$ for $j \in<m>$ and

$$
X_{1} \geq X_{2} \geq \cdots \geq X_{m} \geq 0 \text {. }
$$

Let

$$
P=\left(\begin{array}{llll}
A_{11}^{-1} & & & \\
& A_{22}^{-1} & & \\
& & \ddots & \\
& & & A_{m m}^{-1}
\end{array}\right) .
$$

Then $(P A) Z=0$ and $Z_{i}=-\sum_{j \neq i} A_{i i}^{-1} A_{i j} Z_{j}$ for $i \in<m>$.

Further,

$$
\left\|Z_{i}\right\| \leq \sum_{j \neq i}\left\|A_{i i}^{-1}\right\| \cdot\left\|A_{i j}\right\| \cdot\left\|Z_{j}\right\| \text { for } i \in<m>
$$

Define

$$
\mu(P A)=\left(\begin{array}{cccc}
1 & -\left\|A_{11}^{-1}\right\| \cdot\left\|A_{12}\right\| & \cdots & -\left\|A_{1}^{-1}\right\| \cdot\left\|A_{m}\right\| \\
-\left\|A_{22}^{-1}\right\| \cdot\left\|A_{21}\right\| & 1 & \cdots & -\left\|A_{22}^{-1}\right\| \cdot\left\|A_{2 m}\right\| \\
\vdots & \vdots & \ddots & \vdots \\
-\left\|A_{m}^{-1}\right\| \cdot\left\|A_{m 1}\right\| & -\left\|A_{m m}^{-1}\right\| \cdot\left\|A_{m 2}\right\| & \cdots & 1
\end{array}\right)=K=\left(k_{i j}\right)
$$

Then (1.4) becomes

$$
\mu(P A) X \leq 0,
$$

where $X=\left(X_{1}, X_{2}, \cdots, X_{m}\right) \in R^{m} \backslash\{0\}$.Our aim is to show that the hypotheses of the theorem contradict (1.5).

To do that, it is sufficient to show that, for some $l \in<m>$,

$$
\sum_{i=1}^{l} \sum_{j=1}^{m} k_{i j} X_{j}>0 \text {. }
$$


Let

$y_{1}=X_{1}-X_{2}, y_{2}=X_{2}-X_{3}, \cdots, y_{m-1}=X_{m-1}-X_{m}, y_{m}=X_{m}$

By (1.3),

$$
y \geq 0 \text { (and since } Z \neq 0, y \neq 0 \text { ). }
$$

Define $B(r, s)=\sum_{i=1}^{r} \sum_{j=1}^{s} k_{i j}$. Then (1.6) becomes

$$
\sum_{j=1}^{m} B(l, j) y_{i}>0 \text {. }
$$

By (1.1), there exists $l \leq k$ such that

$$
B(r, m) \leq 0 \text { for } r<l \text {, }
$$

But

$$
B(l, m)>0 .
$$

From (1.2) and (1.9), we infer $B(l, j)>0$ for $j=1,2, \cdots, l-1$.And from (1.10), we infer $B(l, j)>0$ for $j=l, l+1, \cdots, m$.

In fact, if $l=1$, then $B(1,1) \geq B(1,2) \geq \cdots \geq B(1, m)>0$.

If $l>1$, by (1.10), then $B(l, m)=\sum_{i=1}^{l}\left(1-\tilde{R}_{i}(A)\right)>0$.

Further, $B(l, l) \geq B(l, l+1) \geq \cdots \geq B(l, m)>0$.

Since

$$
\begin{aligned}
& B(l, l-1)=\sum_{i=1}^{l} \sum_{j=1}^{l-1} k_{i j}=\sum_{j=1}^{l-1} \sum_{i=1}^{l} k_{i j}=\sum_{j=1}^{l-1} k_{i j}+\sum_{j=1}^{l-1} \sum_{\substack{j=1 \\
j \neq k}}^{l} k_{i j} \\
& =\sum_{j=1}^{l-1} 1-\sum_{j=1}^{l-1} \sum_{i \neq j}^{l}\left\|A_{i i}^{-1}\right\| \cdot\left\|A_{i j}\right\| \geq \sum_{j=1}^{l-1}\left(1-\tilde{C}_{j k}(A)\right),
\end{aligned}
$$

By (1.9), then $B(l-1, m)=\sum_{i=1}^{l-1} \sum_{j=1}^{m} k_{i j}=\sum_{i=1}^{l-1}\left(1-\tilde{R}_{i}(A)\right) \leq 0$.

From (1.2), then $\sum_{i=1}^{l-1}\left(1-\tilde{C}_{i k}(A)\right)>0$.Further,

$B(l, l-1)>0$. Hence

$$
B(l, l-2)>0, \cdots, B(l, 2)>0, B(l, 1)>0 .
$$

Further, (1.11), (1.12) and (1.7) imply (1.8), therefore (1.6), contradicting (1.5). Hence, $A$ is nonsingular.

If we assume that

$$
1<\tilde{R}_{i_{0}}(A) \text { for at lease one } i,
$$

then Theorem 1 can be improved.

Theorem 2. Let $A=\left(a_{i j}\right) \in C^{n \times n}$ be partitioned as (1) and satisfy (2.1), in which $A_{i i}$ is nonsingular for $i \in<m>$.If there exists $k \in<m>$ such that

$$
\left|S_{k}\right|=k \text { implies } \sum_{i \in S_{k}}\left(1-\tilde{R}_{i}(A)\right) \geq 0 \text { for all } S_{k} \subseteq<m>
$$

and (1.6) holds. Then $A$ is nonsingular.
Proof. We will call the index $t$ a skip if $y_{t}>0$, and SK the set of skips (which we know to be nonempty). Our object is to show that, for suitable $l \in\langle m\rangle$, $B(l, t) \geq 0$ for all $t \in S K$,

and

$B(l, t)>0$ for at least one $t \in S K$.

This will prove (1.8) and establish a contradiction of the suppostition that $A$ is singular.

Observe first that, by (2.2),

$$
B(r, m) \geq 0 \text { if } r \geq k \text {. }
$$

Next, let $r$ be the smallest index in SK. Firstly, we show the case $r \geq k$.

If $B(r, m)>0$, then

$$
B(r, r) \geq B(r, r+1) \geq \cdots \geq B(r, m)>0 .
$$

Let $l=r$, then $B(l, t)=B(r, t)>0$ for all $t \in S K$.

Hence (2.3) and (2.4) hold, and we are done. So suppose $B(r, m)=0$. Because $r$ is the first skip, we know

$$
X_{1}=X_{2}=\cdots=X_{r}=\max \left\{X_{i}\right\} \text {. }
$$

Hence $1 \leq R_{i}(A)$ for all $i \in\langle r\rangle$. Since (2.1) and $r \geq k$, there exists $S_{k}$ such that $\sum_{i \in S_{k}}\left(1-R_{i}(A)\right)<0$, contradicting (2.2).

Secondly we show the case $r<k$. Let $l$ be the smallest skip such that $B(l, m)$ is nonnegative.

If there exists $l$, by (2.2), then $l<k$. Further $t \leq l$ and $B(t, m) \leq 0$ for $t \in S K$. So $t \leq \sum_{i=1}^{t} R_{i}(A)$. By (1.2), then

$$
B(l, t)=\sum_{i=1}^{l} \sum_{\substack{j=1 \\ j \neq i}}^{t} k_{i j}=\sum_{j=1}^{t} k_{j j}+\sum_{j=1}^{t} \sum_{\substack{i=1 \\ i \neq j}}^{l} k_{i j} \geq \sum_{j=1}^{t}\left(1-\tilde{C}_{i k}(A)\right)>0 .
$$

Hence (2.4) holds. Since

(2.3) holds.

$$
B(l, l) \geq B(l, l+1) \geq \cdots \geq B(l, m) \geq 0,
$$

If $l$ does not exist, then $B(t, m)<0$ for all $t \leq k$ and $t \in S K$. Further $t<\sum_{i=1}^{t} \tilde{R}_{i}(A)$. Let $l=k$, by (2.2), then $B(k, m) \geq 0$. Hence $B(k, t) \geq B(k, m) \geq 0$. For all $t \geq k$ and $t \in S K$, by (1.2), then

$$
B(k, t)=\sum_{i=1}^{k} \sum_{\substack{j=1 \\ j \neq i}}^{t} k_{i j}=\sum_{j=1}^{t} k_{j j}+\sum_{j=1}^{t} \sum_{\substack{i=1 \\ i \neq j}}^{k} k_{i j} \geq \sum_{j=1}^{t}\left(1-\tilde{C}_{i k}(A)\right)>0 .
$$

Hence (2.3) and (2.4) hold. The theorem is true.

\section{APPLICATIONS}

Corollary 3. Let $A=\left(a_{i j}\right) \in C^{n \times n}$ be partitioned as (1), in which $A_{i i}$ is nonsingular for $i \in<m>$.If $A$ and $k$ satisfy (1.2) or satisfy (2.1) and (2.2), and for some $\alpha, 0<\alpha<1$, 
$1>\alpha \tilde{R}_{i}(\mathrm{~A})+(1-\alpha) \tilde{C}_{i k}(\mathrm{~A})$ for all $i \in<m>$. Then $A$ is nonsingular.

Corollary 4. Let $A=\left(a_{i j}\right) \in C^{n \times n}$ be partitioned as (1), in which $A_{i i}$ is nonsingular $M-$ matrix for $i \in<m>$. Take the vector norm to be Euclidean norm. If $A$ and $k$ satisfy (1.1) and (1.2), then $A$ is positive stable matrix.

Proof. For any $\lambda \in \sigma(A)$, there exists a nonzero

$$
X=\left(X_{1}^{T}, X_{2}^{T}, \cdots X_{M}^{T}\right)
$$

such that $A X=\lambda X$, in which the partitioned vector of $X$ is uniform with $A$.

If $\operatorname{Re} \lambda \leq 0$, then $\lambda \notin \bigcup_{j \in<m>} \sigma\left(\mathrm{A}_{j j}\right)$ (see [6]). Further,

(see [6]).

$$
\frac{\left\|\left(\mathrm{A}_{i i}-\lambda I_{n_{i}}\right)^{-1} y\right\|}{\|y\|} \leq \frac{\left\|\mathrm{A}_{i i}^{-1}\right\| \cdot\|y\|}{\|y\|} \text { for all } y \in C^{n} \backslash\{0\}
$$

Hence $\left\|\left(\mathrm{A}_{i i}-\lambda I_{n_{i}}\right)^{-1}\right\| \leq\left\|\mathrm{A}_{i i}^{-1}\right\|$. For $A_{\lambda}=A-\lambda I$, then $\tilde{R}_{i}(\mathrm{~A}) \geq \tilde{R}_{i}\left(\mathrm{~A}_{\lambda}\right), \tilde{C}_{i k}(\mathrm{~A}) \geq \tilde{C}_{i k}\left(\mathrm{~A}_{\lambda}\right)$. Therefore, $\sum_{i \in S_{k}}\left(1-\tilde{R}_{i}\left(\mathrm{~A}_{\lambda}\right)\right) \geq \sum_{i \in S_{k}}\left(1-\tilde{R}_{i}(\mathrm{~A})\right)>0$,

$|S|>\min \left\{\sum_{i \in S} \tilde{R}_{i}(\mathrm{~A}), \sum_{i \in S} \tilde{C}_{i k}(\mathrm{~A})\right\} \geq \min \left\{\sum_{i \in S} \tilde{R}_{i}\left(\mathrm{~A}_{\lambda}\right), \sum_{i \in S} \tilde{C}_{i k}\left(\mathrm{~A}_{\lambda}\right)\right\}$.

By Theorem $1, A_{\lambda}=A-\lambda I$ is nonsingular, contradicting $A_{\lambda}$ to be singular. Hence the theorem is true.
Similarly, we can get the following corollary.

Corollary 5. Let $A=\left(a_{i j}\right) \in C^{n \times n}$ be partitioned as (1), in which $A_{i i}$ is nonsingular $M-$ matrix for $i \in<m>$. Take the vector norm to be Euclidean norm. If $A$ and $k$ satisfy (1.6),(2.1) and (2.2), then $A$ is the positive stable matrix.

Clearly, the above results have generalized the corresponding results of [1] [4].

\section{ACKNOWLEDGMENT}

We thank the reviewers for their valuable comments and suggestions on this paper.

\section{REFERENCES}

[1] Brualdi R A. Mellendorf S., "Regions in the complex plane containingthe eigenvalues of a matrix, " Amer. Math. Monthly, vol. 101, pp. 975-985, December 1994.

[2] V. N. Solov'ev, “A generalization of Gershgorin's theorem,” Math. USSR Izvestiya, vol. 23, pp. 545-560, October 1984.

[3] V. A. Pupkov, "An isolated eigenvalue of a matrix and the structure of its eigenvector,” USSR Comput. Math. Phys., vol. 23, pp. 14-20, October 1983.

[4] Hoffman, "Gersgorin variations I: on a theme of Pupkov and Solov'ev,” Linear Algebra Appl., vol. 304, pp. 173-177, January 2000.

[5] R.Horn and C.Johnson, "Matrix Analysis," Cambridge University Press, Cambridge, 1985.

[6] A.Berman, R.J.Plemmons, "Nonnegative Matrices in the Mathematical Sciences,” SIAM Press, Philadelphia, 1994.

[7] M. Li and Q.C. Li, "New Criteria for Nonsingular $H$-matrices," Chinese journal of engineering mathematics, vol. 29, pp.715-719, October 2012. 